\title{
Characterisation of the Shape of Microparticles via Fractal and Fourier Analyses of Scanning Electron Microscope Images
}

\author{
Volodymyr V. Kindratenko, Pierre J. M. Van Espen, \\ Boris A. Treiger and René E. Van Grieken \\ Micro- and Trace Analysis Center (MiTAC), Department of Chemistry, \\ University of Antwerpen (UIA), B-2610 Antwerpen, Belgium
}

\begin{abstract}
Two different approaches based on Fourier and fractal analyses, are applied for particle shape characterisation. The advantages and limitations of both approaches as well as their fields of application are discussed. Examples of the application of these approaches for particles of various types are given.
\end{abstract}

Key words: shape, fractal dimension, Fourier analysis, particle characterisation, image analysis.

\section{Introduction}

Computer controlled scanning electron microscopy (CC SEM) in combination with energydispersive X-ray microanalysis (EDX) has proven to be a powerful tool for analysing microscopic particles. The method provides both compositional (via EDX spectra) and morphological (via SEM images) characteristics of particles. In practice, the chemical composition is used as the main source of information, e.g. to classify the particles according to their origin. However, there are situations where the shape of the particles is of primary importance. For example, the shape and size of so-called 'T-grain' silver halide crystals determine the performance of the photographic emulsion [1]. Therefore, the ability to classify the crystals based on their shapes is of interest for evaluating photographic materials. As another example, the shape of some atmospheric microparticles is indicative for their origin, e.g. two types of aerosol particles, spherical fly ash and irregularly shaped soil dust particles, originate from different processes, but have similar compositions [2,3]. Again, only the shape of such microparticles allows us to differentiate between them and to make source apportionment.

The problem is how to reveal and represent the shape in a form suitable for particle characterisation. At the current stage of shape analysis one can distinguish a large number of characterisation methods, the most commonly applied being 1) shape factors based [4], 2) chain coding ([5], p. 205), 3) Fourier analysis ([5], p. 206) and 4) fractal analysis ([5], p. 162). Here we examine the applicability of fractal analysis for describing the shape of individual aerosol particles and Fourier analysis for describing of the shape of silver halide crystals.

\section{Method}

\section{Preprocessing of SEM images}

SEM images consist of pixels where the intensity (colour) of each pixel is proportional to the number of the back-scattered electrons, emitted from the corresponding point at the surface of a material. Some of them represent the corresponding points of the microparticles (foreground pixels), the others represent background. In the present study we used images with 256 grey levels. For the extraction of the shape of particles only foreground pixels are of interest. In order to separate foreground from background pixels, the thresholding technique [6] was applied. A binary image is obtained with only two intensity

\footnotetext{
${ }^{*}$ To whom correspondence should be addressed
} 
values. In such images the shape information is contained in the particle edge. The border following algorithm [7] was used for finding and storing a connected list of coordinates of the particle edge. Thus, the shape (silhouette edge) is defined as the list of the connected coordinates of the particle edge.

\section{Description of particle shape using Fourier coefficients}

The particle's silhouette edge can be described in polar coordinates as a function of radius $\boldsymbol{R}$ vs. the sweep angle $\boldsymbol{\theta}$ from the center of gravity of the particle [8]. This function can be expanded in a Fourier series:

$$
R(\theta)=R_{0}+\sum_{n=1}^{\infty}\left(A_{n} \cos (n \theta)+B_{n} \sin (n \theta)\right)
$$

where $\boldsymbol{R}_{\mathbf{0}}$ is the radius of a circle having the same area as the profile, generated by the particle's silhouette edge; $\boldsymbol{\theta}$ is the polar angle measured from an arbitrary reference line; $\boldsymbol{A}_{\mathbf{n}}$ and $\boldsymbol{B}_{\mathbf{n}}$ are the Fourier series coefficients. Each term of this series is called a harmonic. Beddow [9] proposed to use the harmonic amplitude $\boldsymbol{A}_{\mathbf{n}}{ }^{2}+\boldsymbol{B}_{\mathbf{n}}{ }^{2}$ that indicates the contribution of the $\boldsymbol{n}$-th component in the overall shape where $\boldsymbol{n}$ is the harmonic order. The more complicated the shape is, the more harmonics should be taken into account for the description of the shape. Only the first few are responsible for the 'general' shape and they can be used for the purpose of identification [8]. E.g., for a particle with a pronounced hexagonal shape, only the first six harmonics are important (Fig. 1B); a particle with a pronounced triangular shape can be reconstructed quite well by the first six coefficients too (Fig. 1C).

Mostly convex shapes can be described correctly by this method. In the other case it is possible to obtain different radii for the same angle. Some extensions of this techniques for the case of particles with a concave shape can be found in the literature [10].

\section{Description of particle shape using Fractal dimension}

Fractal dimension is a concept to describe the measure of the 'irregularity' of irregular or selfsimilar shapes. Self-similarity means that any part of the shape looks the same as any other part and they all look the same as the whole structure. Real particles are not fractal from a mathematical point of view because self-similarity cannot be observed over the entire range of sizes - only over some limited intervals. Also, a particle can have several intervals where one can observe the self-similarity. Different intervals correspond to so-called 'textural' and 'structural' fractal dimensions [11].

The concept of fractal dimension can be applied to particle characterisation in the following way. The perimeter $\boldsymbol{P}$ of the contour of a particle depends on the length $\boldsymbol{L}$ of the 'yardstick' with which we make the measurements: $\boldsymbol{P} \sim \boldsymbol{L}^{\boldsymbol{\alpha}}$. The fractal dimension of the contour of the particle in this case is $\mathbf{1 - \alpha}$. Thus, the calculation of the fractal dimension is based on the estimation of the length of the silhouette edge at different resolutions and the fractal dimension is obtained from a graph in which the logarithm of the length estimate is plotted vs. the logarithm of the resolution (Richardson plot) [12]. A 'Hybrid' method [13] for fractal dimension calculation is used in this work. Measurements were performed with a 'yardstick' from 0.02 to 0.2 of the maximal distance between points of the silhouette edge of the microparticles (maximum Feret diameter).

There are difficulties with the exact determination of the fractal dimension for computerised images. A circle has definitely no fractality, but we obtained a textural fractal dimension equal to 1.01 for a computerised image of a circle. This phenomenon is explained by the discrete nature of the image: any distance measure on a regularly spaced grid will reflect the finite dimension of the grid. The simplest way to take this error into account is to add the absolute value of the slope of the Richardson plot for the image of the circle to the error in the fractal dimension determination of the experimental particles.

\section{Experimental}

The measurements were performed on a JEOL JSM-6300 SEM controlled by a PGT IMIX system using an electron energy of $20 \mathrm{keV}$ and a beam current of ca. $1 \mathrm{nA}$ and a typical magnification of $5000 x$. Details about the samples and their preparation can be found in $[1,14]$. Image processing is done with software written by the authors. 


\section{Results and Discussion}

\section{Silver halide crystals}

An example of the results of the Fourier analysis of the silver halide crystals (Fig. 1A) is given in Fig. 1D. The three different classes of crystals can be clearly defined based on analysis of only the third and sixth harmonics. Thus, for the triangular shaped crystals the 3rd harmonic has the largest amplitude while for the hexagonal shaped crystals the 6th harmonic has the largest amplitude. Overlapping crystals can be revealed by the second harmonic. Small crystals usually have a large amplitude of the first harmonic, but they can be eliminated from the analysis using geometrical parameters such as the radius of the equivalent circle.

The analysis results for the silver halide crystals are summarised in Fig. 2. Nine crystals of triangular shape, 13 crystals of irregular hexagonal shape, 13 crystals of perfect hexagonal shape and 10 sets of overlapping crystals were analysed; the average values and standard deviations of the second, the third and the sixth coefficients are shown.

\section{Aerosol particles}

A typical example of a spherical fly ash particle, its EDX spectrum and the results of the fractal analysis are presented in Fig. 3A. A backscattered electron image of a soil dust particle, its EDX spectrum and the results of fractal analysis are shown in Fig. 3B. Comparison of Figs 3A and 3B leads to the conclusion that it is impossible to judge whether a particle belongs to the fly ash class or the soil dust class solely on the base of the composition. At the same time, fractal analysis reveals that spherically shaped fly ash particles possess no fractality, whereas the 'textural' fractality of soil dust particle is evident.

The results of the analysis of 72 individual aerosol particles are summarised in Table 1 . The mean value of the fractal dimension of the soil dust particles is 1.04 with a standard deviation of 1.01. This value is statistically different from the average value of $1.01 \pm 0.01$ obtained for 35 fly ash particles. The absence of fractality for the spherically shaped fly ash particles and the presence of only textural fractality for soil dust particles holds for the all particles analysed.

\section{Conclusion}

Two algorithms for shape description of individual particles were tested on real samples. The results obtained indicate that they can be used for the correct classification of the particles.

Fourier description is very attractive for particles with regular shapes, especially for those with axes of symmetry. Normally, the first 6 to 8 Fourier coefficients reproduce the shape quite well. Classification based on the fractal dimension is more suitable for particles with pronounced irregular contours.

\section{References}

[1] I. Geuens, Development of Micro and Surface Analysis Methods for the Quantitative Characterization of Silver Halide Microcrystals, Ph.D. thesis, University of Antwerp, 1993.

[2] A.R. Ramsden, M. Shibaoka, Atmospheric Environment, 1982, 16, pp. 2191 - 2206.

[3] G.L. Fisher, B.A. Prentice, D. Silberman, J.M. Ondov, A.H. Blermann, R.C. Ragaini, A.R. McFariand, Environ. Sci. Technol., 1978, 12, pp. 447 - 451.

[4] A.I. Medalia, Powder Technol., 1970/71, 4, pp. 117 - 138.

[5] J.C. Russ, Computer-Assisted Microscopy: The Measurement and Analysis of Images, Plenum Press: New York, 1990, pp. 205, 206, 162.

[6] A.D. Brink, Pattern Recognit. Lett., 1989, 9, pp. 335-341.

[7] A. Rosenfeld, A.C. Kak, Digital Picture Processing, second edition, Academic Press, Inc.: London, 1982, vol. 2, p. 219.

[8] B. Raeymaekers, P. Van Espen and F. Adams, Microchim. Acta, 1984, II, pp. 437-454.

[9] J.K. Beddow, G.C. Philip, A.F. Vetter, M.D. Nasta, Powder Technol., 1977, 18, pp. 19-25.

[10] J.K. Beddow (ed.), Particle Characterization in Technology, vol. 2: Morphological Analysis, CRC Press: Boca Raton, Florida, 1984, pp. 3-14. 
[11] B. Kaye, A Random Walk Through Fractal Dimensions, VCH Publishers: New York, 1989.

[12] B.B. Mandelbrot, Science, 1967, 156, pp. 633-638.

[13] N.N. Clark, Powder Technol., 1986, 46, pp. 45 - 52.

[14] V.V. Kindratenko, P.J. Van Espen, B.A. Treiger, R.E. Van Grieken, Environ. Sci. Technol., 1994, 28, pp. $2194-2202$.

Table 1. Fractal dimension and standard deviation for different types of individual aerosol particles.

\begin{tabular}{|c|c|c|}
\hline Type of aerosol particle & $\begin{array}{c}\text { Number of particles } \\
\text { studied }\end{array}$ & $\begin{array}{c}\text { Textural fractal } \\
\text { dimension }\end{array}$ \\
\hline spherically shaped fly ash & 35 & $1.01 \pm 0.01$ \\
\hline soil dust & 37 & $1.04 \pm 0.01$ \\
\hline
\end{tabular}



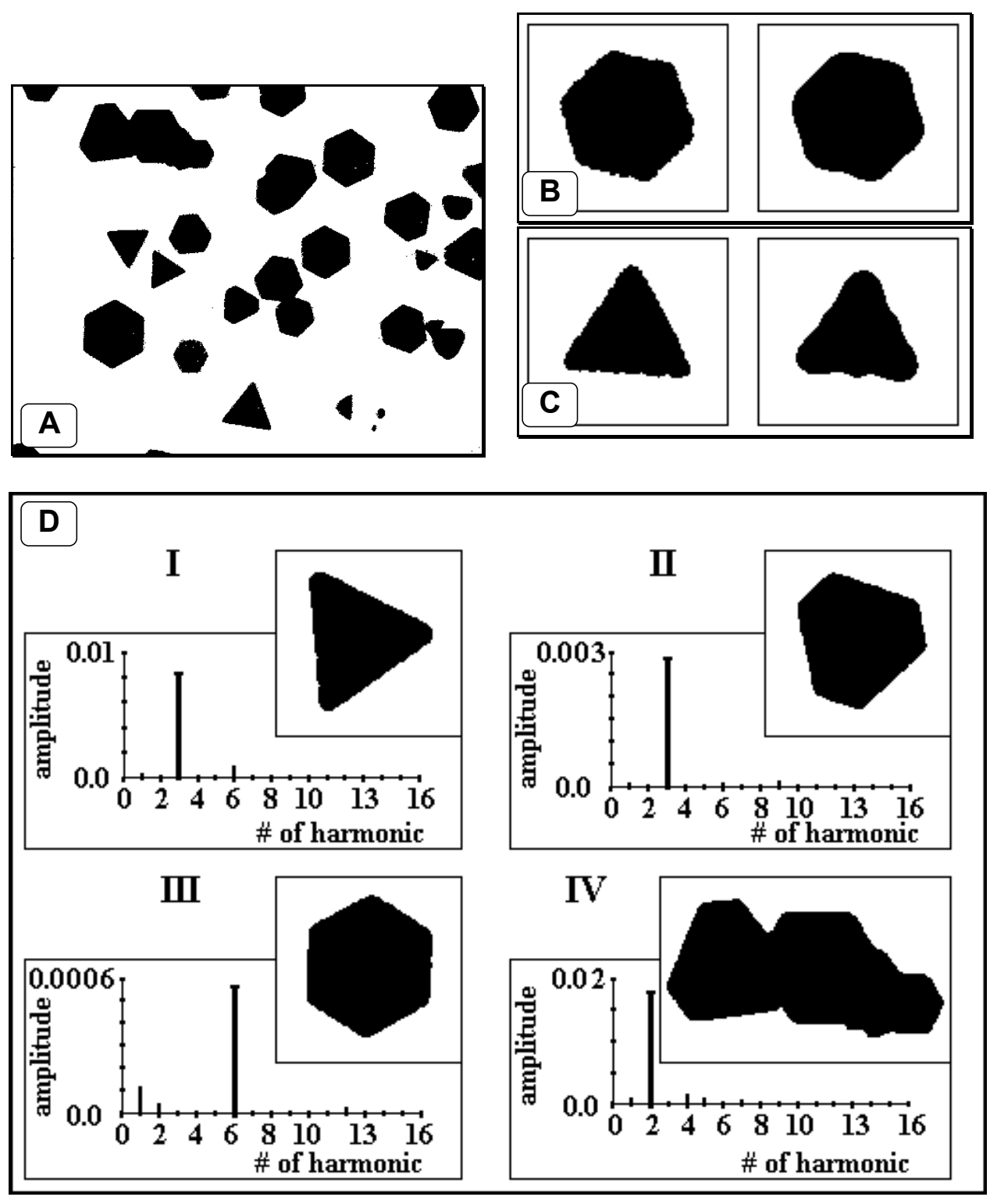

Figure 1. A. 'T-grain' silver halide crystals (SEM backscattered electron image); B. The hexagonally shaped silver halide crystal (left) and restored hexagon by using the first 6 Fourier coefficients (right); C. The triangular silver halide crystal (left) and restored triangle by using the first 6 Fourier coefficients (right); D. Amplitude of the Fourier coefficients for different crystal shape. 


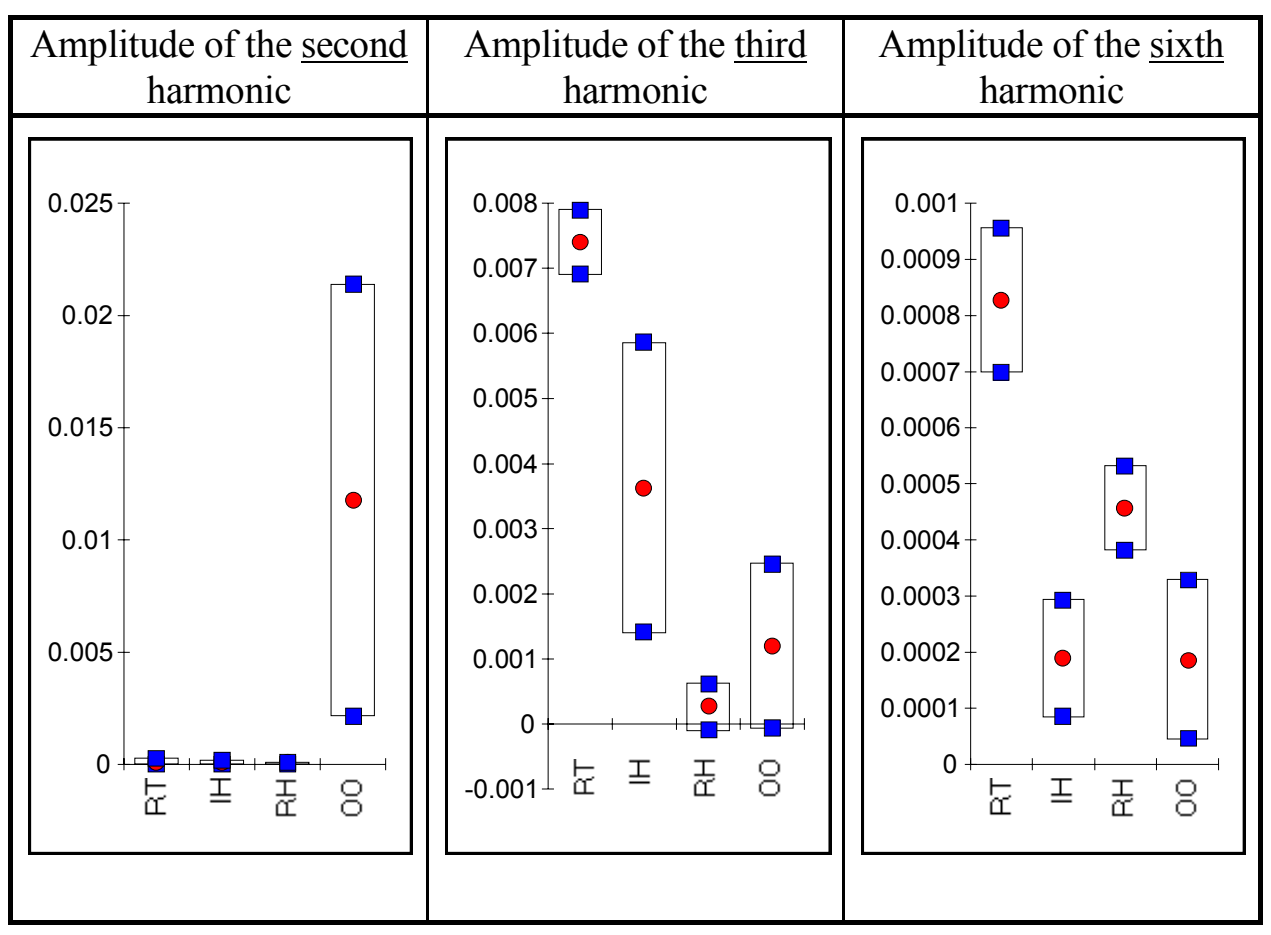

Figure 2. Average values of the amplitude and standard deviation of the second, third and sixth harmonics for the four different types of silver halide crystals (RT - triangles, IH - irregular hexagons, RH - hexagons, OO - overlapping crystals).

Figure 3. A. Spherically shaped fly ash particle (SEM backscattered electron image, EDX spectrum and results of the fractal analysis); B. Soil dust particle (SEM backscattered electron image, EDX spectrum and results of the fractal analysis). The following designations are used: $\mathbf{r}$ - the coefficient of correlation for the given regression straight line, FD - fractal dimension. The yardstick is expressed in units of the maximum Feret diameter. 


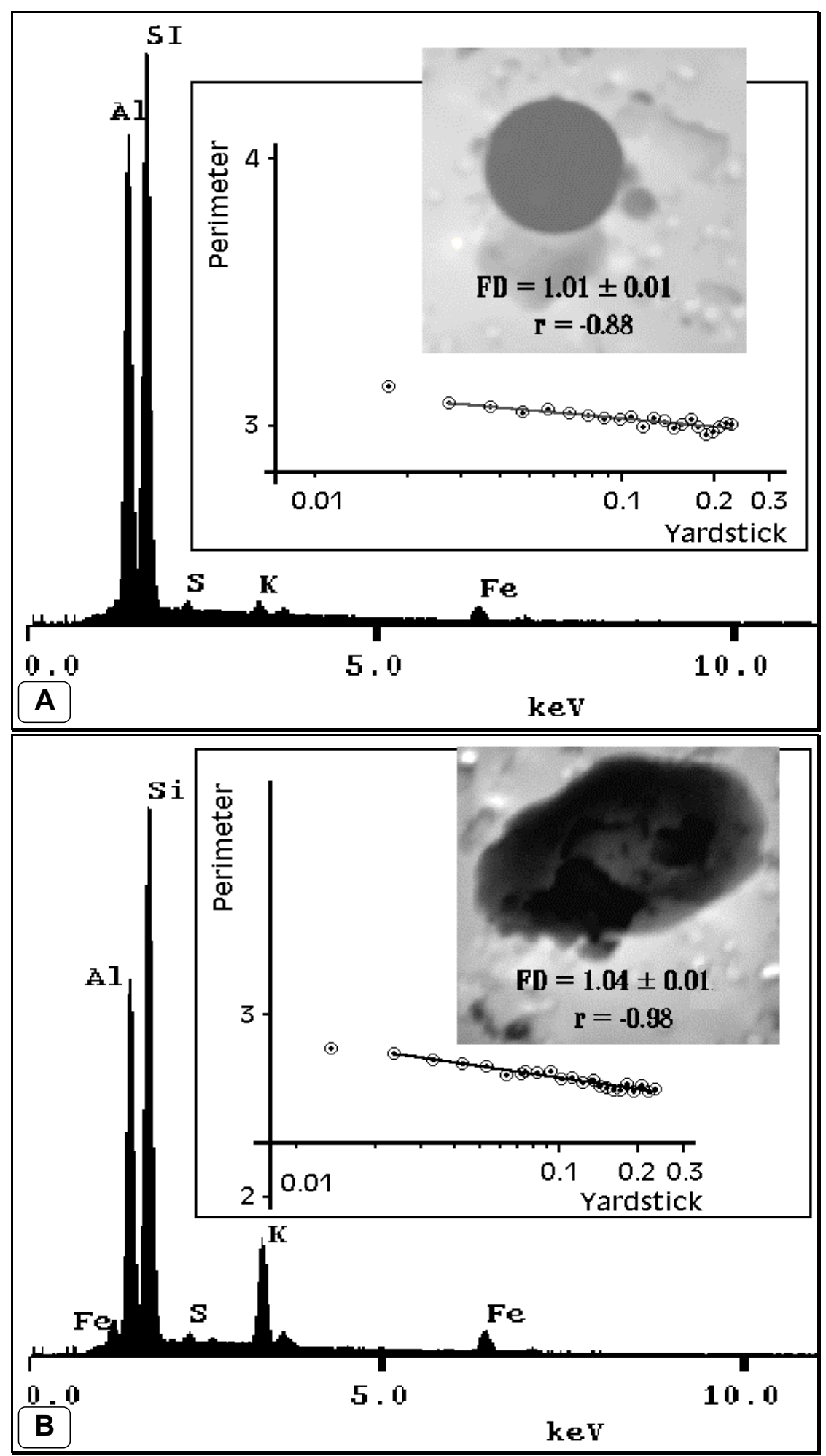

